\title{
A REPRESENTATION RESULT FOR TWO-INPUT TWO-OUTPUT DECENTRALIZED CONTROL SYSTEMS
}

\author{
DAVID J. CLEMENTS
}

(Received 23 June 1978)

(Revised 5 September 1978)

\begin{abstract}
This paper studies the decentralized control and stabilization of two-input, two-output finite dimensional linear systems. A representation result for the system and a characterization of all stabilizing controllers are given in terms of certain fixed polynomial matrices and a stability constraint.
\end{abstract}

\section{Introduction}

In the control of a number of physical systems the natural controller structure is decentralized, the system is for some reason divided into a number of subsystems with the control of the total system being achieved by the control of each of the subsystems. However, even though the controllers are local, their design should ideally be determined on a system-wide basis.

There has not been a great deal of analytical work on decentralized systems carried out, though the amount is increasing. Perhaps the most significant result known is contained in the paper of Wang and Davison [2] where it is shown that a necessary and sufficient condition for the existence of a decentralized controller (in fact, a proper decentralized controller) to stabilize the system is that the fixed polynomial be stable. (These results are only applicable to finite dimensional linear systems with finite dimensional linear controllers.) A great deal more work is needed to understand the role of the fixed polynomial in the structure of the plant and also what limitations, if any, it imposes on permissible controllers. The paper [1] studies certain aspects of these questions, thereby extending the results of [2]. 
One problem of interest is the explicit parameterization of the class of all decentralized controllers which stabilize the system, for such a parameterization may possibly form the basis of some optimal design procedure.

In this paper a general two-input two-output plant is studied with respect to its stabilizability by decentralized controllers. It is shown that if the plant is stabilizable, there exists a decentralized controller which stabilizes the plant by moving all of the non-fixed modes to $\infty$, so that the only modes of the closed-loop system are the fixed modes. The resulting closed-loop system is patently unsuitable for physical implementation; however, for theoretical purposes it is important. This leads to a complete description of the class of all stabilizing decentralized controllers in terms of this improper controller and two arbitrary diagonal polynomial matrices satisfying a stability constraint.

In Section 2, the relevant material of [2] is reviewed together with some results on polynomial matrix representations of transfer functions and, finally, conditions for the closed-loop stability of the system. In Section 3, the implications of the stabilizability of the plant on the polynomial matrix representations of the plant and the existence of an improper controller are considered. Then in Section 4, a parameterization of the class of stabilizing controllers is developed.

\section{Review}

Let $P(s)$ be a $p \times m$ rational matrix, and let $A(s)$ and $B(s)$ be $p \times p$ and $p \times m$ polynomial matrices respectively such that

$$
P(s)=A(s)^{-1} B(s) \text {. }
$$

Then the pair $[A(s), B(s)]$ is called a left factorization of $P(s)$.

Next, the pair $[A(s), B(s)]$ is said to be relatively left prime if there exist polynomial matrices $X(s)$ and $Y(s)$ with dimensions $p \times p$ and $m \times p$ respectively such that

$$
A(s) X(s)+B(s) Y(s)=I_{p}, \quad \text { the } p \times p \text { identity matrix. }
$$

Further, it can be shown that this is equivalent to the greatest common divisor of all of the $p \times p$ minors of the augmented matrix $\left[\begin{array}{lll}A(s) & B(s)\end{array}\right]$ being unity.

Some other results needed are:

1. If $P(s)=A(s)^{-1} B(s)=A_{1}(s)^{-1} B_{1}(s)$ and $[A(s), B(s)]$ is a relatively left prime pair of polynomial matrices, then there exists a $p \times p$ polynomial matrix $V(s)$ such that $A_{1}(s)=V(s) A(s)$ and $B_{1}(s)=V(s) B(s)$. If $\left[A_{1}(s), B_{1}(s)\right]$ is also a relatively left prime pair then $V(s)$ is unimodular, that is, it has constant nonzero determinant. 
2. For any $m \times p$ polynomial matrix $K(s)$, the pair $[A(s)+B(s) K(s), B(s)]$ is relatively left prime if and only if $[A(s), B(s)]$ is a relatively left prime pair.

3. If $P(s)=\bar{A}(s)^{-1} \bar{B}(s)$ and $[\bar{A}(s), \bar{B}(s)]$ is not a relatively left prime pair, then there exists a nonsingular polynomial matrix $U(s)$ and a relatively left prime pair $[A(s), B(s)]$ such that $\bar{A}(s)=U(s) A(s), \bar{B}(s)=U(s) B(s)$ and $P(s)=A(s)^{-1} B(s)$. In this case det $U(s)$ is called the hidden polynomial and the zeros of $\operatorname{det} U(s)$ are called the hidden modes of $P(s)$.

The transfer matrix $P(s)$ is strictly proper (proper) if $P(\infty)=0(P(\infty)$ is a finite matrix). Otherwise it is called improper. A polynomial is termed Hurwitz or stable if all of its zeros are in the open left half of the complex plane.

The following is a restatement of the main result of [2].

THEOREM 1. Suppose $P(s)$ is a strictly proper transfer matrix with the input vector divided into l disjoint sets and the output divided into l disjoint sets. Then there exists a proper decentralized controller $C(s)$ stabilizing the system if and only if the fixed polynomial $f(s)$ is Hurwitz, where

$$
f(s)=\text { g.c.d. }\{\operatorname{det}[A(s)+B(s) K]: K \in \mathscr{K}\}
$$

and $\mathscr{K}$ is the class of all constant block diagonal matrices consistent with the partitioning of the inputs and outputs.

REMARKS.

1. In [2], the plant $P(s)$ is defined by

$$
P(s)=H^{\prime}(s I-F)^{-1} G
$$

for constant matrices $H, F$ and $G$ of appropriate dimensions and the fixed modes of $P(s)$ are then defined by

$$
\text { g.c.d. }\left\{\operatorname{det}\left(s I-F+G K H^{\prime}\right): K \in \mathscr{K}\right\} \text {. }
$$

It is then a simple matter to show that if $\left(H^{\prime}, F, G\right)$ is a minimal realization of $P(s)$ in the sense that $(F, G)$ is a completely controllable pair and $\left(H^{\prime}, F\right)$ is a completely observable pair, and if $(A(s), B(s))$ is a relatively left prime pair of polynomial matrices satisfying $P(s)=A^{-1}(s) B(s)$ then this definition of fixed modes coincides with that given in Theorem 1.

2. For $P(s)$ either proper or improper, Theorem 1 remains true. However, if $P(s)$ is improper, there is no guarantee that there exists a proper controller $C(s)$ which not only stabilizes the system but also makes the closed-loop system proper. As the following example shows, there exist improper $P(s)$ such that for all proper decentralized controllers the closed-loop system is improper. 


\section{Example}

Let

and let

$$
P(s)=(s-1)^{-1}\left[\begin{array}{ll}
1 & s^{3} \\
1 & 1
\end{array}\right]
$$

$$
C(s)=\left[\begin{array}{cc}
\frac{g_{1}(s)}{f_{1}(s)} & 0 \\
0 & \frac{g_{2}(s)}{f_{2}(s)}
\end{array}\right],
$$

where $\operatorname{deg} g_{i}(s) \leqslant \operatorname{deg} f_{i}(s)$ for $i=1,2$. Then the fixed polynomial is $f(s)=1$. A little analysis then shows that the closed-loop transfer matrix $(I+P(s) C(s))^{-1} P(s)$ is improper for any proper decentralized controller $C(s)$.

3. Remark 2 contrasts with the situation for centralized controllers where the following holds. For any plant $P(s)$, there exists a proper centralized controller $C(s)$ such that the closed-loop system is proper and stable, provided the plant $P(s)$ has no uncontrollable and/or unobservable modes.

\section{Structure of two-input two-output system}

Let $P$ be a $2 \times 2$ rational matrix written as

$$
P=p^{-1}\left[\begin{array}{ll}
p_{11} & p_{12} \\
p_{21} & p_{22}
\end{array}\right],
$$

where $p$ is the McMillan polynominal for $P$. (The argument for polynomials is deleted unless it is specifically needed.) Further let (A, B) be any relatively left prime pair of polynominal matrices such that

and where

$$
P=A^{-1} B
$$

$$
A=\left[\begin{array}{ll}
a_{11} & a_{12} \\
a_{21} & a_{22}
\end{array}\right], \quad B=\left[\begin{array}{ll}
b_{11} & b_{12} \\
b_{21} & b_{22}
\end{array}\right] .
$$

Any decentralized controller for the plant $P$ is represented as a diagonal rational matrix $C_{d}$. Write $C_{d}=B_{d} A_{d}^{-1}$ where $\left(A_{d}, B_{d}\right)$ is a relatively right prime pair of diagonal polynomial matrices with

$$
A_{d}=\left[\begin{array}{cc}
g_{11} & 0 \\
0 & g_{22}
\end{array}\right], \quad B_{d}=\left[\begin{array}{cc}
l_{11} & 0 \\
0 & l_{22}
\end{array}\right] .
$$


The relative right primeness of the pair $\left(A_{d}, B_{d}\right)$ is clearly equivalent to

$$
\left(g_{11}, l_{11}\right)=1 \text { and }\left(g_{22}, l_{22}\right)=1 \text {. }
$$

If the decentralized controller $C_{d}$ is applied to the plant $P$, the rational matrix $P_{c}$ representing the closed-loop transfer matrix of the controlled plant is $P_{c}=\left[I+P C_{d}\right]^{-1} P$ which can then be written as

$$
P_{c}=A_{d}\left[A A_{d}+B B_{d}\right]^{-1} B
$$

From (2) and (3) the plant $P$ can be represented by

$$
P=A^{-1} B=d_{12}^{-1}\left[\begin{array}{cc}
-d_{23} & -d_{24} \\
d_{13} & d_{14}
\end{array}\right],
$$

where $d_{i j}$ is the $2 \times 2$ minor formed from columns $i$ and $j$ of the augmented polynomial matrix $D=\left[\begin{array}{ll}A & B\end{array}\right]$. Then comparison of (1) and (6) and recognition of the fact that $d_{12}=\operatorname{det}(A)=p$ (because $(A, B)$ is a relatively left prime pair) implies that (6) is identical to (1). The representation (6) is used in the remainder of this section.

Next, taking determinants of both sides of (6) and noting that $d_{12}=\operatorname{det}(A)$ and $d_{34}=\operatorname{det}(B)$, there follows the important relationship

$$
d_{13} d_{24}=d_{12} d_{34}+d_{14} d_{23} \text {. }
$$

Then, using $P_{c}=\left[I+P C_{d}\right]^{-1} P$ in conjunction with (6) and (7) or using (5) with the definitions of $d_{11}, d_{12}$, etc., the closed-loop transfer matrix becomes

$$
P_{c}=p_{c}^{-1}\left[\begin{array}{cc}
g_{11}\left(-d_{23} g_{22}+d_{34} l_{22}\right) & -g_{11} d_{24} g_{22} \\
g_{11} d_{13} g_{22} & \left(d_{14} g_{11}+d_{34} l_{11}\right) g_{22}
\end{array}\right]
$$

with

$$
p_{c}=d_{12} g_{11} g_{22}-d_{23} l_{11} g_{22}+d_{14} l_{22} g_{11}+d_{34} l_{11} l_{22} \text {. }
$$

The representation (8) is a general representation of the transfer matrix for the controlled plant for any decentralized controller.

Next, an explicit definition of the fixed polynomial for decentralized control is needed. Setting $g_{11}=1, g_{22}=1, l_{11}=k_{11}$ and $l_{22}=k_{22}$, (9) becomes

$$
p_{K}=d_{12}-d_{23} k_{11}+d_{14} k_{22}+d_{34} k_{11} k_{22}
$$

with $p_{K}$ denoting $p_{c}$ for the special case of a constant decentralized controller $K=\operatorname{diag}\left\{k_{11}, k_{22}\right\}$. Then, according to [2], the fixed polynomial $f$ for decentralized 
control is

$$
\begin{aligned}
f & =\text { g.c.d. }\left\{\operatorname{det}(A+B K): K=\operatorname{diag}\left\{k_{11}, k_{22}\right\}\right\} \\
& =\text { g.c.d. }\left\{d_{12}-d_{23} k_{11}+d_{14} k_{22}+d_{34} k_{11} k_{22}: k_{11}, k_{22}\right\} \\
& =\text { g.c.d. }\left\{d_{12}, d_{23}, d_{14}, d_{34}\right\} .
\end{aligned}
$$

This contrasts with the fixed polynomial for centralized control (that is, the polynomial of the unobservable and/or uncontrollable modes) which is 1 because (A, B) is relatively left prime pair. Equivalently,

$$
\text { g.c.d. }\left\{d_{12}, d_{23}, d_{14}, d_{34}, d_{13}, d_{24}\right\}=1 \text {, }
$$

this being a simple restatement of the relative left primeness of the pair $(A, B)$ in terms of the $2 \times 2$ minors of $D=\left[\begin{array}{ll}A & B\end{array}\right]$.

\section{REMARKS.}

(i) Note that the 2 off-diagonal terms $d_{13}$ and $d_{24}$ in (6) do not appear explicitly in (10); though they do implicitly via $d_{34}$ and the relationship (7).

(ii) The closed-loop matrix transfer function for a constant decentralized controller $K$ [from (8)] is

$$
p_{K}=p_{K}^{-1}\left[\begin{array}{cc}
-d_{23}+d_{34} k_{11} & -d_{24} \\
d_{13} & d_{14}+d_{34} k_{22}
\end{array}\right]
$$

indicating that the 2 off-diagonal numerator terms are unaffected by the constant feedback.

From (10) and (7), it is clear that $f^{2}$ divides $d_{13} d_{24}$, the product of the off-diagonal numerator terms of $P$. However, more than this can be shown.

LEMMA 1. Given (7), (10) and (11), there exist coprime polynomials $f_{1}$ and $f_{2}$ such that

(i) $f=f_{1} f_{2}$,

(ii) $f_{1}^{2}$ divides $d_{13}$,

(iii) $f_{2}^{2}$ divides $d_{24}$.

Proof. (See Appendix A.)

This lemma enables the fixed polynomial part of the closed-loop transfer matrix $P_{c}$ to be separated from $P_{c}$ in a prescribed manner as follows.

Define the polynomials $d_{13}^{\prime \prime}$ and $d_{24}^{\prime \prime}$ by

$$
d_{13}=f_{1}^{2} d_{13}^{\prime \prime}, \quad d_{24}=f_{2}^{2} d_{24}^{\prime \prime}
$$


and define the polynomials $\tilde{d}_{12}, \tilde{d}_{23}, \tilde{d}_{14}$ and $\tilde{d}_{34}$ by

$$
\begin{array}{ll}
d_{12}=f \tilde{d}_{12}, & d_{23}=f \tilde{d}_{23}, \\
d_{14}=f \tilde{d}_{14}, & d_{34}=f \tilde{d}_{34} .
\end{array}
$$

These polynomials are well defined by Lemma 1 and the definition of $f$. Finally, set

$$
F=\left[\begin{array}{cc}
f_{1} & 0 \\
0 & f_{2}
\end{array}\right]
$$

Then, (8) can be written as

where

$$
P_{c}=\tilde{p}_{c}^{-1} F^{-1} A_{d} N F,
$$

$$
\begin{gathered}
N=\left[\begin{array}{cc}
-\tilde{d}_{23} g_{22}+\tilde{d}_{34} l_{22} & -d_{24}^{\prime \prime} g_{22} \\
d_{13}^{\prime \prime} g_{11} & \tilde{d}_{14} g_{11}+\tilde{d}_{34} l_{11}
\end{array}\right], \\
\tilde{p}_{c}=\tilde{d}_{12} g_{11} g_{22}-\tilde{d}_{23} l_{11} g_{22}+\tilde{d}_{14} l_{22} g_{11}+\tilde{d}_{34} l_{11} l_{22} .
\end{gathered}
$$

Further, since $F$ and $A_{d}$ are diagonal they commute. Therefore it is sensible to consider the pair of polynomial matrices $(F, N F)$.

LEMMA 2. The pair $(F, N F)$ is a relatively left prime pair of polynomial matrices if and only if $\left(f_{1}, g_{22}\right)=1$ and $\left(f_{2}, g_{11}\right)=1$.

\section{Proof. (See Appendix B.)}

An immediate observation is that the pair $(F, N F)$ is relatively left prime for any constant decentralized controller $K$ for in this case $g_{11}=g_{22}=1$.

In [2], it is shown that for any decentralized controller, the fixed polynomial is a divisor of the McMillan polynomial of the closed-loop system. The next lemma shows that there exists a decentralized controller for which the fixed polynomial equals the McMillan polynomial of the closed-loop system and, moreover, that for the resulting numerator matrix $N$, the pair $(F, N F)$ is relatively left prime.

LEMMA 3. There exists a choice of $l_{11}, g_{11}, l_{22}$ and $g_{22}$ such that

$$
\begin{aligned}
p_{c} & =f, \quad\left(\text { that is } \tilde{p}_{c}=1\right) \\
\left(f, g_{22}\right) & =1, \\
\left(f, g_{11}\right) & =1 .
\end{aligned}
$$


We denote this controller pair by $X_{d}$ and $Y_{d}$, where

$$
X_{d}=\left[\begin{array}{cc}
g_{11} & 0 \\
0 & g_{22}
\end{array}\right], \quad Y_{d}=\left[\begin{array}{cc}
l_{11} & 0 \\
0 & l_{22}
\end{array}\right] .
$$

Proof. (See Appendix C.)

With $X_{d}$ and $Y_{d}$ as defined in Lemma 3, it follows that

$$
A X_{d}+B Y_{d}=F,
$$

where the polynomial matrix $F$ satisfies

$$
\operatorname{det}(F)=f
$$

This can be checked by either straightforward evaluation of the determinant of $A X_{d}+B Y_{d}$ or by recognizing that in (8), $p_{\mathrm{c}}=\operatorname{det}\left(A A_{d}+B B_{d}\right)$. Now from (5) it follows that

$$
P_{c}=X_{d} F^{-1} B .
$$

However, from Lemma 3, (15) becomes

$$
P_{c}=X_{d} F^{-1} N F,
$$

where $\tilde{p}_{c}=1$ and the fact that $X_{d}$ and $F$ commute have been used. Therefore the pairs $(F, B)$ and $(F, N F)$ are left polynomial factorizations of the same rational matrix $X_{\vec{d}}^{-1} P_{c}$. From Lemmas 2 and $3,(F, N F)$ is a relatively left prime pair, so there exists a polynomial matrix $V$ such that

$$
\begin{aligned}
& B=V N F, \\
& F=V F .
\end{aligned}
$$

From (17) and the definition of $F, \operatorname{det}(V)=1$ so $V$ is a unimodular matrix.

Substitute (18) in (16) to give $A X_{d}+V N F Y_{d}=V F$. Then noting that $F$ and $Y_{d}$ commute this becomes

$$
A X_{d}=V\left[N Y_{d}-I\right] F \text {. }
$$

In Appendix $C, X_{d}$ is chosen so that the pair $\left(X_{d}, F\right)$ is relatively left (and right) prime. Thus, there exists a polynomial matrix $M$ such that

$$
A=V M F
$$

and so, collecting the above results,

$$
M X_{d}+N Y_{d}=I .
$$


In centralized control theory, if $(A, B)$ is a polynomial pair such that $P=A^{-1} B$ then there exists a relatively right prime pair $\left(A_{c}, B_{c}\right)$ such that

$$
A A_{c}+B B_{c}=H,
$$

where $H$ is the greatest common left divisor of $A$ and $B$. Then dividing out $H$, we obtain

$$
\bar{A} A_{c}+\bar{B} B_{c}=I
$$

for the relatively left prime pair $(\bar{A}, \vec{B})$, and $d=\operatorname{det}(H)$ is the fixed polynomial of the plant. Therefore, (20) might be thought of as an extension of this result.

As is well known, $H$ can be constructed by column operations on the matrix $\left[\begin{array}{ll}A & B\end{array}\right]$. That is, there exists a unimodular matrix $U$ such that

$$
\left[\begin{array}{ll}
A & B
\end{array}\right] U=\left[\begin{array}{ll}
H & 0
\end{array}\right] \text {. }
$$

Then $U$ is partitioned as

$$
U=\left[\begin{array}{ll}
A_{c} & \hat{B}_{c} \\
B_{c} & \hat{A}_{c}
\end{array}\right]
$$

so that

$$
A \hat{B}_{c}+B \hat{A_{c}}=0 .
$$

Now consider again the decentralized control case. It can be shown (see Appendix D) that there exist diagonal polynomial matrices $\hat{X}_{d}$ and $\hat{Y}_{d}$ which are relatively left prime and satisfy

$$
M \hat{Y}_{d}+N \hat{X}_{d}=G
$$

where $G=\left[\begin{array}{cc}0 & d_{24}^{\prime \prime} \\ d_{13}^{\prime \prime} & 0\end{array}\right]$. Moreover, the polynomial matrix $W=\left[\begin{array}{cc}X_{d} & \hat{Y}_{d} \\ Y_{d} & \hat{X}_{d}\end{array}\right]$ is unimodular. Therefore,

$$
\left[\begin{array}{ll}
A & B
\end{array}\right] W=V\left[\begin{array}{ll}
F & G F
\end{array}\right]
$$

so that, in the sense that the right side of (22) contains the fixed quantities of $P,(22)$ is an extension of (21).

\section{Parameterization of all stable controllers}

Given the structural results of the previous section it is relatively straightforward to parameterize all decentralized controllers $C_{d}$ which stabilize the plant $P$. The parameterization presented here may be thought of as an extension of the results 
in [3, Lemma 3] where all stabilizing controllers for a plant are parameterized in terms of an arbitrary stable transfer matrix.

Let $C_{d}=B_{d} A_{\bar{d}}^{-1}$ be a decentralized controller that stabilizes the plant $P$. Then the closed-loop transfer matrix is

$$
P_{c}=A_{d}\left(A A_{d}+B B_{d}\right)^{-1} B
$$

and $d=\operatorname{det}\left(A A_{d}+B B_{d}\right)$ is stable.

From (22),

$$
\left[\begin{array}{ll}
A & B
\end{array}\right]\left[\begin{array}{ll}
X_{d} & \hat{Y}_{d} \\
Y_{d} & \hat{X}_{d}
\end{array}\right]=V\left[\begin{array}{ll}
F & G F
\end{array}\right]
$$

where $X_{d}, Y_{d}, \hat{Y}_{d}$ and $\hat{X}_{d}$ are all diagonal and the composite matrix formed from them is unimodular. It is then not difficult to show that

$$
\left[\begin{array}{ll}
A & B
\end{array}\right]=V\left[\begin{array}{ll}
F & G F
\end{array}\right]\left[\begin{array}{ll}
T_{11} & T_{12} \\
T_{21} & T_{22}
\end{array}\right],
$$

where each of $T_{11}, T_{12}, T_{21}$ and $T_{22}$ is diagonal and the composite matrix so formed is unimodular. Therefore with the controller $\left(A_{d}, B_{d}\right)$,

$$
A A_{d}+B B_{d}=V\left[\begin{array}{ll}
F & G F
\end{array}\right]\left[\begin{array}{c}
S_{1} \\
S_{2}
\end{array}\right],
$$

where $S_{1}$ and $S_{2}$ are diagonal matrices with $S_{1}=T_{11} A_{d}+T_{12} B_{d}$ and $S_{2}=T_{21} A_{d}+T_{22} B_{d}$. Also note that the various diagonal matrices commute. Then,

and so

$$
A A_{d}+B B_{d}=V\left[S_{1}+G S_{2}\right] F
$$

$$
\left(A A_{d}+B B_{d}\right)^{-1}=F^{-1} s^{-1}\left(\tilde{S}_{1}-G S_{2}\right) V^{-1},
$$

where $s=\operatorname{det}\left(S_{1}+G S_{2}\right)$ and $\tilde{S}_{1}$ is simply $S_{1}$ with the diagonal elements interchanged. Taking determinants of both sides of (23) gives $h=s f$, and $s$ is stable. However, $\operatorname{det}\left(S_{1}+G S_{2}\right)$ equals $\operatorname{det}\left(\tilde{S_{1}}-G S_{2}\right)$. Thus, altogether, there exist diagonal matrices $\tilde{S}_{1}$ and $S_{2}$ such that

$$
\operatorname{det}\left(\tilde{S}_{1}-G S_{2}\right)=\operatorname{det}\left(\tilde{S}_{1}\right)-\operatorname{det}(G) \operatorname{det}\left(S_{2}\right)
$$

is stable.

Conversely suppose that there is given $\tilde{S}_{1}$ and $S_{2}$ diagonal such that $\operatorname{det}\left(\widetilde{S}_{1}\right)-\operatorname{det}(G) \operatorname{det}\left(S_{2}\right)$ is stable. Then define

$$
\begin{aligned}
& A_{d}=\left(X_{d} \tilde{S}_{1}+\tilde{Y}_{d} S_{2}\right), \\
& B_{d}=\left(Y_{d} \tilde{S}_{1}+\mathscr{X}_{d} S_{2}\right) .
\end{aligned}
$$


Thus $A_{d}, B_{d}$ are diagonal, and relatively prime provided $\tilde{S}_{1}$ and $S_{2}$ are relatively prime and

$$
A A_{d}+B B_{d}=V\left(\tilde{S}_{1}+G S_{2}\right) F
$$

and $\operatorname{det}\left(A A_{d}+B B_{d}\right)$ is stable, provided $f$ is. This completes the characterization of all stabilizing decentralized controllers.

It is thus apparent that in the study of two-input two-output systems the two fixed matrices $F$ and $G$ play a central role. The matrix $F$ contains information about the modes of the plant which are invariant with respect to the class of all decentralized controllers. The matrix $G$ contains information about the crosscoupling zeros of the plant and, as the above analysis shows, these zeros are directly related to the stability constraint.

Finally, recall that in Remark 2 in Section 2 it is noted that there exist improper plants which cannot be made proper by any decentralized controller. It is not clear whether the above analysis is of any help in determining precisely when this situation may occur.

\section{Conclusions}

This paper presents a detailed analysis of decentralized controllers for a twoinput two-output finite dimensional linear plant. In particular, a structural result involving the fixed polynomial and certain fixed off-diagonal terms is given. This structural result is then used to parameterize all decentralized controllers that stabilize the plant, when the fixed polynomial is stable.

The major question arising from this paper is whether these results can be extended to more general systems. There are two directions in which these results might be extended. The first is to the case of $l$ inputs and $l$ outputs with each output being used to control only one input. Probably, the methods of this paper are applicable to this case though the analysis would quickly become prohibitive. The second possible direction of research is the case of two vector inputs and two vector outputs. Here it appears that the methods used in this paper, with their reliance on individual polynomials, are not suitable for such a generalization. A new characterization of the $F$ matrix would be a major requirement. Finally, the connections, if any, between the results of this paper and the ideas in [1] need to be explained.

\section{Acknowledgement}

The work described in this paper was supported by the Australian Research Grants Committee. 


\section{Appendix A}

Proof of Lemma 1. First, suppose that $d_{13} d_{24}=0$. If both $d_{13}$ and $d_{24}$ are zero, then $f=1$ and the lemma holds for $f_{1}=1$ and $f_{2}=1$. Now assume $d_{13} \neq 0$. Then $\left(f, d_{13}\right)=1$, so set $f_{1}=1$ and $f_{2}=f$. Again the lemma holds. Similarly, if $d_{24} \neq 0$, set $f_{1}=f$ and $f_{2}=1$.

Second, assume $d_{13} d_{24} \neq 0$. If $f=1$, set $f_{1}=1$ and $f_{2}=1$. If $f$ is not constant, let $\alpha$ be any non-trivial irreducible factor of $f$. Then $\alpha^{2}$ divides $d_{13} d_{24}$. This then implies that either $\alpha^{2}$ divides $d_{13}$ or $\alpha^{2}$ divides $d_{24}$. For, if not, then $\alpha$ divides $d_{13}, d_{24}$ and $f$ which contradicts the fact that g.c.d. $\left\{d_{13}, d_{24}, f\right\}=1$. Consequently, $f$ can be written as $f_{1} f_{2}$ for some polynomials $f_{1}$ and $f_{2}$ where $f_{1}^{2}$ divides $d_{13}$ and $f_{2}^{2}$ divides $d_{24}$. Finally, $f_{1}$ and $f_{2}$ must be coprime otherwise there exists a non-trivial irreducible polynomial which divides $d_{13}, d_{24}$ and $f$, which is again a contradiction. This completes the proof of the lemma.

Remark. It is obvious that if $d_{13}^{\prime \prime}$ and $d_{24}^{\prime \prime}$ are defined by $d_{13}=f_{1}^{2} d_{13}^{\prime \prime}$ and $d_{24}=f_{2}^{2} d_{24}^{\prime \prime}$, then $\left(f_{1}, d_{24}^{\prime \prime}\right)=1$ and $\left(f_{2}, d_{13}^{\prime \prime}\right)=1$.

\section{Appendix B}

Proof of Lemma 2.

$$
[F, N F]=\left[\begin{array}{cccc}
f_{2} & 0 & f_{1}\left(-\tilde{d}_{23} g_{22}+\tilde{d}_{34} l_{22}\right) & -f_{2} d_{24}^{\prime \prime} g_{22} \\
0 & f_{2} & f_{1} d_{13}^{\prime \prime} g_{11} & f_{2}\left(\tilde{d}_{14} g_{11}+\tilde{d}_{24} l_{11}\right)
\end{array}\right],
$$

which is column equivalent to

$$
\left[\begin{array}{cccc}
f_{1} & 0 & 0 & f_{2} d_{24}^{\prime \prime} g_{22} \\
0 & f_{2} & f_{1} d_{13}^{\prime \prime} g_{11} & 0
\end{array}\right] .
$$

Thus, $(F, N F)$ is a relatively left prime pair if and only if $\left(f_{1}, f_{2} d_{24}^{\prime \prime} g_{22}\right)=1$ and $\left(f_{2}, f_{1} d_{13}^{\prime \prime} g_{11}\right)=1$. From Lemma $1,\left(f_{1}, f_{2}\right)=1$ and from the remark in Appendix $\mathrm{A}$, $\left(f_{1}, d_{24}^{\prime \prime}\right)=1$ and $\left(f_{2}, d_{13}^{\prime \prime}\right)=1$. Therefore, the pair $(F, N F)$ is relatively left prime if and only if $\left(f_{1}, g_{22}\right)=1$ and $\left(f_{2}, g_{11}\right)=1$. This proves the lemma.

\section{Appendix C}

Proof of Lemma 3. Recall that

and so

$$
\tilde{p}_{c}=\tilde{d}_{12} g_{11} g_{22}-\tilde{d}_{23} l_{11} g_{22}+\tilde{d}_{14} l_{22} g_{11}+\tilde{d}_{34} l_{11} l_{22}
$$

$$
\tilde{p}_{c}=\left(d_{12} g_{11}-d_{23} l_{11}\right) g_{22}+\left(d_{14} g_{11}+d_{34} l_{11}\right) l_{22} \text {. }
$$


Then, there exist $g_{22}$ and $l_{22}$ such that $\tilde{p}_{c}=1$ if and only if there exist $g_{11}$ and $l_{11}$ such that $\tilde{d}_{12} g_{11}-\tilde{d}_{23} l_{11}$ and $\tilde{d}_{14} g_{11}+d_{34} l_{11}$ are coprime.

For the moment assume that the polynomial $\tilde{d}_{12} \tilde{d}_{34}+\tilde{d}_{14} \tilde{d}_{23}$ is not identically zero, and note that this is equivalent to $d_{13}^{\prime \prime} d_{24}^{\prime \prime}$ not being identically zero. Then, in this case, the polynomials $\tilde{d}_{12}-\tilde{d}_{23} k_{11}$ and $\tilde{d}_{14}+\tilde{d}_{34} k_{11}$ are coprime for all but a finite number of values of the constant $k_{11}$. To see this, suppose that for a given $k_{11}$, there exists $\tilde{s}$ such that $d_{12}(\bar{s})-\tilde{d}_{23}(\tilde{s}) k_{11}=0$ and $\tilde{d}_{14}(\bar{s})+\tilde{d}_{34}(\tilde{s}) k_{11}=0$. Then $\bar{s}$ must be a zero of the polynomial $d_{12} \tilde{d}_{34}+\tilde{d}_{14} \tilde{d}_{23}$ which is not identically zero by hypothesis. Thus, there are at most a finite number of $\bar{s}$ for which the polynomials $\tilde{d}_{12}-\tilde{d}_{23} k_{11}$ and $\tilde{d}_{14}+\tilde{d}_{34} k_{11}$ can fail to be coprime. However, for each such $\bar{s}$, the corresponding $k_{11}$ is uniquely defined by $k_{11}=\tilde{d}_{12}(\bar{s}) / \tilde{d}_{23}(\bar{s})$ and/or

$$
k_{11}=-\tilde{d}_{14}(\bar{s}) / \tilde{d}_{34}(\bar{s}) \text {. }
$$

This follows because $\tilde{d}_{23}(\bar{s})$ and $\tilde{d}_{34}(\bar{s})$ cannot both be zero, otherwise a contradiction to g.c.d. $\left\{\tilde{d}_{12}, \tilde{d}_{23}, \tilde{d}_{14}, \tilde{d}_{34}\right\}=1$ is obtained.

Now assume that $\tilde{d}_{12} \tilde{d}_{34}+\tilde{d}_{14} \tilde{d}_{23}=0$. Then choose $g_{11}$ and $l_{11}$ such that $d_{12} g_{11}-\tilde{d}_{23} l_{11}=\left(\tilde{d}_{12}, \tilde{d}_{23}\right)$. [In general, neither $g_{11}$ nor $l_{11}$ will be constants in contrast to the case $\tilde{d}_{12} \hat{d}_{34}+\tilde{d}_{14} \tilde{d}_{23} \neq 0$.] Define $\hat{d}_{12}$ and $\hat{d}_{23}$ by $\hat{d}_{12}=\left(d_{12}, d_{23}\right) \hat{d}_{12}$ and $\tilde{d}_{23}=\left(\tilde{d}_{12}, \tilde{d}_{23}\right) \hat{d}_{23}$. Then $\hat{d}_{12} \tilde{d}_{34}+\tilde{d}_{14} \hat{d}_{23}=0$ and since, $\left(d_{12}, d_{23}\right)=1$, this implies that $\tilde{d}_{14}=\beta \hat{d}_{12}$ and $\tilde{d}_{34}=-\beta \hat{d}_{23}$ for some polynomial $\beta$. Therefore,

$$
\tilde{d}_{14} g_{11}+\tilde{d}_{34} l_{11}=\beta\left(\hat{d}_{12} g_{11}-\hat{d}_{23} l_{11}\right)=\beta \text {. }
$$

However, $\beta$ and $\left(\tilde{d}_{12}, \tilde{d}_{23}\right)$ are coprime; otherwise, there exists a common factor of $\tilde{d}_{12}, \tilde{d}_{34}, \tilde{d}_{14}$ and $\tilde{d}_{23}$. Finally, there exist $g_{12}$ and $l_{22}$ such that $\left(\tilde{d}_{12}, d_{23}\right) g_{22}+\beta l_{22}=1$. Again, $g_{22}$ and $l_{22}$ are generally not constant polynomials.

The proof of the lemma is then a simple consequence of the following

LEMMA C1. Let $p$ and $q$ be two real polynomials which are coprime, and let $e$ be any fixed polynomial. Then there exist polynomials $r$ and $t$ such that $p r+q t=1$ and $(e, t)=1$.

Proof. Suppose $\bar{r}$ and $\bar{t}$ are any polynomials satisfying $p \bar{r}+q \bar{t}=1$. Then if $p r+q t=1$, it follows that for some polynomial $\beta, r=\bar{r}+\beta q$ and $t=\bar{t}-\beta p$. Now since $(\bar{t}, p)=1$, it is clear that there exists a constant $\beta$ such that $\bar{t}\left(s_{0}\right)-\beta p\left(s_{0}\right) \neq 0$ for all $s_{0}$ satisfying $e(s)=0$. This proves Lemma $\mathrm{Cl}$.

To complete the proof of Lemma 3, use Lemma $\mathrm{Cl}$ to choose $g_{11}$ and $g_{22}$ each coprime with $f$. 


\section{Appendix D}

Define $A=V^{-1} A$ and $\bar{B}=V^{-1} B$. Then from the choice of $g_{11}$ and $l_{11}$ in Appendix $\mathrm{C}$ and noting that $\bar{A} X_{d}+\bar{B} Y_{d}=F$, there follows

$$
\left[\begin{array}{ll}
\tilde{a}_{11} & \tilde{b}_{11} \\
\tilde{a}_{21} & b_{\partial 1}
\end{array}\right]\left[\begin{array}{l}
g_{11} \\
l_{11}
\end{array}\right]=\left[\begin{array}{c}
f_{1} \\
0
\end{array}\right],
$$

where

$$
A=\left[\begin{array}{ll}
\bar{a}_{11} & \bar{a}_{12} \\
\tilde{a}_{21} & \bar{a}_{22}
\end{array}\right], \quad \bar{B}=\left[\begin{array}{ll}
b_{11} & b_{12} \\
b_{21} & b_{22}
\end{array}\right] .
$$

Since $\left(g_{11}, l_{11}\right)=1$, there exist $g_{12}$ and $l_{12}$ such that

$$
\left[\begin{array}{ll}
g_{11} & g_{12} \\
l_{11} & l_{12}
\end{array}\right]
$$

is unimodular with determinant equal to 1 . Thus

$$
\left[\begin{array}{ll}
\tilde{a}_{11} & \bar{b}_{11} \\
\tilde{a}_{21} & \bar{b}_{21}
\end{array}\right]\left[\begin{array}{ll}
g_{11} & g_{12} \\
l_{11} & l_{12}
\end{array}\right]=\left[\begin{array}{cc}
f_{1} & n_{11} \\
0 & n_{12}
\end{array}\right]
$$

for some $n_{11}$ and $n_{22}$. Taking determinants of both sides of this equation gives $d_{13}=f_{1} n_{12}$ and so $n_{12}=f_{1} d_{13}^{\prime \prime}$. However, since $\widetilde{B}=N F$ and $\bar{A}=M F$, then $f_{1}$ divides $\bar{a}_{11}$ and $\bar{b}_{11}$ and hence $f_{1}$ divides $n_{11}$. Then finally,

$$
\left[\begin{array}{ll}
\tilde{a}_{11} & b_{11} \\
\tilde{a}_{21} & b_{21}
\end{array}\right]\left[\begin{array}{ll}
g_{11} & g_{12}-\bar{n}_{11} g_{11} \\
l_{11} & l_{12}-\bar{n}_{11} l_{11}
\end{array}\right]=\left[\begin{array}{cc}
f_{1} & 0 \\
0 & f_{1} d_{13}^{\prime \prime}
\end{array}\right],
$$

where $n_{11}=f_{1} \bar{n}_{11}$. With $l_{33}=g_{12}-\bar{n}_{11} g_{11}$ and $g_{33}=l_{12}-\bar{n}_{11} l_{11}$, the matrix

$$
\left[\begin{array}{ll}
g_{11} & l_{33} \\
l_{11} & g_{33}
\end{array}\right]
$$

is unimodular.

Similarly, there exist $g_{44}$ and $l_{44}$ such that

$$
\left.\left.\left[\begin{array}{ll}
\tilde{a}_{21} & b_{21} \\
\hat{a}_{22} & b_{22}
\end{array}\right]\right] \begin{array}{ll}
g_{22} & l_{44} \\
l_{22} & g_{44}
\end{array}\right]=\left[\begin{array}{cc}
0 & f_{2} d_{24}^{\prime \prime} \\
f_{2} & 0
\end{array}\right]
$$

and again

$$
\left[\begin{array}{ll}
g_{22} & l_{44} \\
l_{22} & g_{44}
\end{array}\right]
$$


is unimodular. Thus,

$$
\left[\begin{array}{llll}
\bar{a}_{11} & \bar{b}_{11} & \bar{a}_{12} & \bar{b}_{12} \\
\bar{a}_{21} & \bar{b}_{21} & \bar{a}_{22} & \bar{b}_{22}
\end{array}\right]\left[\begin{array}{cccc}
g_{11} & l_{33} & 0 & 0 \\
l_{11} & g_{33} & 0 & 0 \\
0 & 0 & g_{22} & l_{44} \\
0 & 0 & l_{22} & g_{44}
\end{array}\right]=\left[\begin{array}{cccc}
f_{1} & 0 & 0 & f_{2} d_{24}^{\prime \prime} \\
0 & f_{1} d_{13}^{\prime \prime} & f_{2} & 0
\end{array}\right]
$$

and rearranging rows and columns:

where

$$
\left[\begin{array}{ll}
A & \bar{B}
\end{array}\right]\left[\begin{array}{cccc}
g_{11} & 0 & l_{33} & 0 \\
0 & g_{22} & 0 & l_{44} \\
l_{11} & 0 & g_{33} & 0 \\
0 & l_{22} & 0 & g_{44}
\end{array}\right]=\left[\begin{array}{cccc}
f_{1} & 0 & 0 & f_{2} d_{24}^{\prime \prime} \\
0 & f_{2} & f_{1} d_{13}^{n} & 0
\end{array}\right]=\left[\begin{array}{ll}
F & G F
\end{array}\right]
$$

$$
G=\left[\begin{array}{cc}
0 & d_{24}^{\prime \prime} \\
d_{13}^{\prime \prime} & 0
\end{array}\right]
$$

Finally $X_{d}, Y_{d}, \hat{X}_{d}$ and $Y_{d}$ are obviously defined to give

$$
\left[\begin{array}{ll}
A & B
\end{array}\right]\left[\begin{array}{ll}
X_{d} & \hat{Y}_{d} \\
Y_{d} & \hat{X}_{d}
\end{array}\right]=V\left[\begin{array}{ll}
F & G F] .
\end{array}\right.
$$

\section{References}

[1] J. P. Corfmat and A. S. Morse, "Decentralized control of linear multivariable systems", Automatica 12 (1976), 479-495.

[2] S. H. Wang and E. J. Davison, "On the stabilization of decentralized control systems", IEEE Trans. Auto. Control AC-18 (1973), 473-478.

[3] D. C. Youla, H. A. Jabr and J. J. Bongiorno, Jr., "Modern Wiener-Hopf design of optimal controllers. Part II: the multivariable case", IEEE Trans. Auto Control AC-21 (1976), 319-338.

School of Electrical Engineering University of New South Wales

Kensington, N.S.W. 2033 